\title{
VDJPipe: a pipelined tool for pre-processing immune repertoire sequencing data
}

\author{
Scott Christley ${ }^{1}$, Mikhail K. Levin², Inimary T. Toby ${ }^{1}$, John M. Fonner ${ }^{3}$, Nancy L. Monson ${ }^{4,5}$, William H. Rounds ${ }^{1}$, \\ Florian Rubelt ${ }^{6}$, Walter Scarborough ${ }^{3}$, Richard H. Scheuermann ${ }^{7,8,9}$ and Lindsay G. Cowell ${ }^{1 *}$
}

\begin{abstract}
Background: Pre-processing of high-throughput sequencing data for immune repertoire profiling is essential to insure high quality input for downstream analysis. VDJPipe is a flexible, high-performance tool that can perform multiple pre-processing tasks with just a single pass over the data files.

Results: Processing tasks provided by VDJPipe include base composition statistics calculation, read quality statistics calculation, quality filtering, homopolymer filtering, length and nucleotide filtering, paired-read merging, barcode demultiplexing, 5' and 3' PCR primer matching, and duplicate reads collapsing. VDJPipe utilizes a pipeline approach whereby multiple processing steps are performed in a sequential workflow, with the output of each step passed as input to the next step automatically. The workflow is flexible enough to handle the complex barcoding schemes used in many immunosequencing experiments. Because VDJPipe is designed for computational efficiency, we evaluated this by comparing execution times with those of PRESTO, a widely-used pre-processing tool for immune repertoire sequencing data. We found that VDJPipe requires $<10 \%$ of the run time required by pRESTO.
\end{abstract}

Conclusions: VDJPipe is a high-performance tool that is optimized for pre-processing large immune repertoire sequencing data sets.

Keywords: Rep-seq, Immune repertoire analysis, Bioinformatics

\section{Background}

The ability to mount an effective immune response and subsequently develop specific immunity relies on the presence of a diverse repertoire of antigen receptors. In jawed vertebrates, the genes encoding antibodies and antigen receptors are somatically generated in lymphocytes through a DNA recombination process, V(D)J recombination, which assembles variable $(\mathrm{V})$, diversity $(\mathrm{D})$, and joining $(\mathrm{J})$ gene segments into genes. The diversity of gene sequences generated by $\mathrm{V}(\mathrm{D}) \mathrm{J}$ recombination is huge (estimated at $>10^{15}$ ) as a result of varying combinations of $\mathrm{V}, \mathrm{D}$, and $\mathrm{J}$ gene segments, as well as sequence modifications (e.g., exonucleolytic trimming and nontemplated nucleotide addition) at the junctions of rearranged gene segments $[1,2]$. In recent years, the increased sample coverage of next generation sequencing technologies has significantly enhanced our ability to

\footnotetext{
* Correspondence: lindsay.cowell@utsouthwestern.edu

'Department of Clinical Sciences, UT Southwestern Medical Center, Dallas, TX 75390, USA

Full list of author information is available at the end of the article
}

obtain detailed characterizations of immune repertoires, as there can easily be more than $10^{6}$ unique sequences for each receptor type $[3,4]$.

Analysis of immune repertoire sequencing data shares some pre-processing tasks with other next generation sequencing data $[5,6]$, but it also has its own unique characteristics, including $5^{\prime}$ and $3^{\prime}$ PCR primer targeting, complex multi-level barcode demultiplexing, and duplicate sequence read collapsing. Table 1 provides a list of immune repertoire analysis tools with their preprocessing capabilities. The set of tools can be divided into three main categories: 1) those providing a workflow from raw data to V, D, and J assignment, 2) those specializing in specific pre-processing operations, and 3) those providing generic pre-processing operations. We excluded web analysis portals such as VDJServer and ARGalaxy, as they provide web-based access to other tools. We indicate each tool's category (1,2 or 3$)$ in Table 1. All of the tools in the first category provide some limited pre-processing capability, though this tends to be restricted to barcode demultiplexing and length 
Table 1 List of analysis tools providing pre-processing functions

\begin{tabular}{|c|c|c|c|c|c|c|c|c|c|c|}
\hline Tool & Category & $\begin{array}{l}\text { Composition } \\
\text { statistics } \\
\text { calculation }\end{array}$ & $\begin{array}{l}\text { Merging } \\
\text { paired-end } \\
\text { reads }\end{array}$ & $\begin{array}{l}\text { Barcode } \\
\text { demultiplexing }\end{array}$ & $\begin{array}{l}\text { PCR primer } \\
\text { removal }\end{array}$ & $\begin{array}{l}\text { Length } \\
\text { filtering }\end{array}$ & $\begin{array}{l}\text { Quality } \\
\text { filtering }\end{array}$ & $\begin{array}{l}\text { Homopolymer } \\
\text { filtering }\end{array}$ & $\begin{array}{l}\text { UMI } \\
\text { consensus } \\
\text { determination }\end{array}$ & $\begin{array}{l}\text { Collapsing } \\
\text { duplicate } \\
\text { reads }\end{array}$ \\
\hline AbStar [16] & 1 & No & Yes & Yes & No & Yes & Yes & No & No & No \\
\hline Decombinator [17] & 2 & No & No & Yes & No & No & No & No & Yes & No \\
\hline Ig-HTS cleaner [18] & 3 & Yes & No & Yes & Yes & Yes & Yes & No & No & No \\
\hline $\begin{array}{l}\text { IgRepertoire } \\
\text { constructor [19] }\end{array}$ & 1 & No & Yes & Yes & No & No & No & No & Yes & No \\
\hline ImmunediveRsity [20] & 1 & No & No & No & No & Yes & Yes & No & No & No \\
\hline iMonitor [21] & 1 & No & Yes & No & No & Yes & Yes & No & No & No \\
\hline IMSEQ [22] & 1 & No & Yes & Yes & No & Yes & Yes & No & No & No \\
\hline MIGEC [7] & 2 & No & Yes & Yes & No & No & Yes & No & Yes & No \\
\hline pRESTO [13] & 3 & No & Yes & Yes & Yes & Yes & Yes & No & Yes & Yes \\
\hline RTCR [23] & 1 & No & No & Yes & No & Yes & Yes & No & No & No \\
\hline VDJPipe & 3 & Yes & Yes & Yes & Yes & Yes & Yes & Yes & No & Yes \\
\hline TCRklass [24] & 1 & No & Yes & No & No & Yes & Yes & No & No & No \\
\hline Abmining [25] & 3 & No & Yes & Yes & No & Yes & Yes & Yes & No & No \\
\hline ClonoCalc [26] & 1 & No & Yes & Yes & No & No & No & No & No & No \\
\hline
\end{tabular}

and quality filtering. Category 2 tools specialize in processing unique molecular identifiers (UMI), which is a strategy to overcome PCR amplification bias and sequencing error [7]. The tools in Category 3, which includes VDJPipe, provide the broadest capability.

VDJPipe is a flexible, high-performance tool for performing a variety of pre-processing operations on immune repertoire sequencing data. VDJPipe efficiently performs all of these pre-processing tasks within a userdefined pipeline that only requires a single pass over large sequencing data files. This has the advantage of significantly decreased processing times, as well as not creating large intermediate files between processing steps, unless desired by the user. VDJPipe is typically the first step in an immunosequencing analysis workflow followed by assignment of V, D, and J gene segments to each sequence with subsequent repertoire annotation and characterization, and it has been successfully applied to analyzing human immune receptor sequences from $B$ and $\mathrm{T}$ cells [8-10].

\section{Implementation}

VDJPipe accepts as input a JSON-formatted configuration file that specifies the set of input sequencing data files and the sequence of processing tasks to be performed on those files. VDJPipe accepts both single-end and paired-end nucleotide sequence read files, and it requires quality scores that can be provided in either a FASTQ file or a FASTA/QUAL file pair. Input sequence files, compressed in gzip or bz2 format, can be read by VDJPipe without being decompressed. Paired-end reads can be initially merged into a single sequence based upon alignment of the overlapping nucleotides, but operations, such as quality filtering, can also be performed on each paired-end read file individually with reads being merged afterward. VDJPipe produces FASTA-formatted output files of processed reads for input into $\mathrm{V}(\mathrm{D}) \mathrm{J}$ assignment software, such as IgBlast.

\section{Sequence read filtering and statistics}

VDJPipe provides many common operations, such as filtering based on nucleotide-level quality and average quality score, ambiguous base calls, homopolymer detection, and sequence length. Each operation has parameters to specify minimum, maximum, or window range values for applying the filter. VDJPipe can calculate various statistics at one or more steps along the processing pipeline. These statistics include nucleotide composition along the length of the read, GC-content histogram, sequence length histogram, average quality score histogram, and base call quality along the length of the read. Generating statistics at multiple points in the pipeline makes it easy to compare how the sequence read composition changes before and after each filtering operation.

\section{Barcode demultiplexing}

It is a common practice with some next generation sequencing studies to multiplex multiple biological samples into a single sequencing run by attaching an identical set of short nucleotide barcodes to all sequences in a given sample. The barcode set is unique for each sample and can be used to demultiplex (split) the combined sequences produced in a single sequencing run back into individual sample files. VDJPipe supports any number of barcodes with its match operation, and 
barcode sequences with their sample identifier are specified in a separate FASTA file. Matching can be performed on the forward or reverse strand, can be limited to a specific search window, and can be a gapped or non-gapped alignment with either a minimal score or a maximum number of mismatches defining a successful match. The match operation uses the standard SmithWaterman local alignment algorithm [11] with a substitution matrix that scores matches and mismatches $(+2$ for match and -2 for mismatch, or 0 for match and +1 for mismatch if only counting mismatches). The matching barcode can be trimmed from the sequence if desired, sequences without a matching barcode can be excluded, and each sequence is tagged with its barcode identifier allowing it to be used in later operations. VDJPipe can handle multiple combinatorial barcodes, such as are used in single-cell sequencing protocols [12], with multiple match operations or with the barcode combinations specified in a CSV file.

\section{$5^{\prime}$ and $3^{\prime}$ primer matching}

Immunosequencing typically uses a targeted PCR protocol with a panel of $5^{\prime}$ ( $\mathrm{V}$ region) and 3' (J or $\mathrm{C}$ region) primers to capture the genes of interest. Other protocols use $5^{\prime}$ RACE, which eliminates the $5^{\prime}$ primer. As with barcodes, VDJPipe's match operation can be used to recognize the primer sequences, trim them from the sequence if desired, and tag each sequence with the primer identifier for use in later operations. Primer sequences are specified in a separate FASTA file.

\section{Duplicate reads}

Adaptive immune cells can undergo clonal expansion which generates daughter cells with identical V(D)J recombination sequences (though some $\mathrm{B}$ cells also undergo somatic hypermutation that can alter the sequence). When sequencing a large number of immune cells, these clones appear as duplicate sequences within the data. Duplicates also appear as a consequence of PCR amplification during sample preparation. Collapsing duplicate reads greatly shrinks the data size and can speed up downstream analyses. However, duplicate read checking in standard tools focused on genome sequencing or RNA-seq assumes only a portion of the sequence needs to be identical in order for the read to be marked as a duplicate [6], but this assumption is invalid for immune repertoire sequencing. Many V, D, and J gene segments are highly similar, and allelic variations present in individuals may only differ by a few nucleotides. Therefore, it is important that the complete read sequence be checked. The standard n-gram hash table approach cannot be used, however, because immune receptor read lengths are typically greater than 250 nucleotides. Thus, VDJPipe utilizes a suffix tree data structure to store the unique sequences found while processing the data. Furthermore, VDJPipe recognizes the sample barcode demultiplexing and collapses duplicate reads within each sample separately. A report of the duplicate count for each read is provided as part of the output.

\section{Results}

We compare the performance of VDJPipe v0.1.7 with that of another software tool specialized for immunosequencing data, pRESTO v0.5.3 [13]. pRESTO has an alternative design of providing a set of Python scripts, each of which performs one step in the pre-processing workflow. For comparison, we use two example data sets provided by pRESTO $[14,15]$ and publically available from SRA under accession ID: ERP003950 and SRX190717. The first data set is Illumina MiSeq $2 \times 250$ stranded paired-end reads from RNA isolated from antibody-secreting mouse cells with primers for the amplification of full-length IgG heavy chain variable regions [14]. The second data set is Roche 454 reads from B-cell RNA isolated from PBMC for human patients across multiple time points before and after exposure to the influenza vaccine [15]. For the first data set, processing steps include merging the paired-end reads into a single read sequence, quality filtering, $5^{\prime}$ and $3^{\prime}$ primer matching, and collapsing duplicate reads. For the second data set, processing steps include length, homopolymer and quality filtering, generating compositional statistics, barcode demultiplexing, $5^{\prime}$ and $3^{\prime}$ primer matching, and collapsing duplicate reads. Together, these two data sets test all the main functions provided by VDJPipe (Table 1). We use the execution scripts provided by pRESTO for the examples but comment out the miscellaneous steps of parsing $\log$ files and compressing intermediate files at the end. The JSON input files used for VDJPipe are provided in the sample_data directory in the source code repository. Tests were run on a quad core Linux computer; VDJPipe only utilizes one processing core, while pRESTO is able to use all four cores.

Tables 2 and 3 show a comparison of execution times for both tools with different size input files for each data set, respectively. We find that VDJPipe is able to complete all processing steps in less than $10 \%$ the time required by pRESTO.

Table 2 Execution times for the Greiff et al., [14] data set

\begin{tabular}{|c|c|c|}
\hline |Sequences| & VDJPipe (1 core) & pRESTO (4 cores) \\
\hline 25,000 & $5.7 \mathrm{~s}(0.1 \mathrm{~s})$ & $1 \mathrm{~m} 43.2 \mathrm{~s}(2.5 \mathrm{~s})$ \\
\hline 250,000 & $1 \mathrm{~m} 7.5 \mathrm{~s}(0.5 \mathrm{~s})$ & 17 m $35.1 \mathrm{~s}(15.6 \mathrm{~s})$ \\
\hline $1,085,869$ & $8 \mathrm{~m} 22.5 \mathrm{~s}(4.6 \mathrm{~s})$ & $89 \mathrm{~m} 14.9 \mathrm{~s}(2 \mathrm{~m} 18.5 \mathrm{~s})$ \\
\hline
\end{tabular}

Execution times are the average of ten runs with the standard deviation in parentheses 
Table 3 Execution times for the Jiang et al., [15] data set

\begin{tabular}{lll}
\hline Sequences & VDJPipe (1 core) & PRESTO (4 cores) \\
\hline 50,000 & $6.5 \mathrm{~s}(0.2 \mathrm{~s})$ & $1 \mathrm{~m} 30.5 \mathrm{~s}(1.2 \mathrm{~s})$ \\
277,826 & $40.3 \mathrm{~s}(0.2 \mathrm{~s})$ & $15 \mathrm{~m} 5.1 \mathrm{~s}(4.9 \mathrm{~s})$ \\
\hline
\end{tabular}

Execution times are the average of ten runs with the standard deviation in parentheses

\section{Conclusion}

VDJPipe is a flexible, high-performance tool for performing a variety of pre-processing operations on immune repertoire sequencing data. Written in $\mathrm{C}++$ and utilizing a pipelined design, VDJPipe can efficiently perform all its operations with just a single pass over the input data file. This results in significantly decreased processing times. This has the additional benefit of not creating large intermediate files (unless desired by the user) between each processing step. Future enhancements include support for multiprocessing and building consensus sequences from unique molecular identifiers.

\section{Availability and requirements}

Project name: VDJPipe

Project home page: https://vdjserver.org/vdjpipe/ index.html

Source code repository: https://bitbucket.org/vdjserver/ vdj_pipe

Docker image: https://hub.docker.com/r/vdjserver/vdj_pipe Operating system(s): Platform independent

Programming language: $\mathrm{C}++$

License: GNU GPL

Any restrictions on use by non-academics: no restrictions. The data sets analyzed in this study are publicly available and described in [14, 15]. The VDJPipe input configuration files used for these two data sets are available in the source code repository in the sample_data directory.

\section{Acknowledgements}

FR would like to thank Mark M. Davis for his advice on this project.

\section{Funding}

This work was supported by an NIAID-funded R01 (Al097403) to LGC. FR was supported, in part, by the Bioinformatics Support Contract (BISC) HHSN272201200028C and the National Institutes of Health, specifically the National Institute of Allergy and Infectious Diseases grant U19A1057229. The funding body had no role in the design of the study or the collection, analysis, and interpretation of data or in writing the manuscript.

\section{Authors' contributions}

SC wrote the manuscript and worked on the software. MKL initially designed and wrote the software. IT conducted the review of analysis tools. JF, NM, WHR, FR, WS, RHS, IT and LGC contributed data, tested the software, and provided feedback on the design. All authors read and approved the final manuscript.

Ethics approval and consent to participate Not applicable.

\section{Consent for publication}

Portions of the abstract were part of a poster abstract presented at Immunology 2016 and originally published in The Journal of Immunology (Copyright 2016 The American Association of Immunologists, Inc., included with permission of The American Association of Immunologists [27]).

\section{Competing interests}

The authors declare that they have no competing interests.

\section{Publisher's Note}

Springer Nature remains neutral with regard to jurisdictional claims in published maps and institutional affiliations.

\section{Author details}

'Department of Clinical Sciences, UT Southwestern Medical Center, Dallas, TX 75390, USA. ${ }^{2}$ Bank of America Corporate Center, Charlotte, NC 28202, USA.

${ }^{3}$ Texas Advanced Computing Center, Austin, TX 78758-4497, USA.

${ }^{4}$ Department of Neurology and Neurotherapeutics, UT Southwestern Medical Center, Dallas, TX 75390, USA. ${ }^{5}$ Department of Immunology, UT

Southwestern Medical Center, Dallas, TX 75390, USA. ${ }^{6}$ Department of Microbiology and Immunology, Stanford University School of Medicine, Stanford, CA 94305, USA. ${ }^{7}$ J. Craig Venter Institute, La Jolla, CA 92037, USA. ${ }^{8}$ Department of Pathology, University of California, San Diego, CA 92093, USA. ${ }^{9}$ La Jolla Institute for Allergy \& Immunology, La Jolla, CA 92037, USA.

Received: 9 March 2017 Accepted: 2 October 2017

Published online: 11 October 2017

References

1. Murugan A, et al. Statistical inference of the generation probability of T-cell receptors from sequence repertoires. Proc Natl Acad Sci U S A. 2012;109(40):16161-6.

2. Casrouge $A$, et al. Size estimate of the alpha beta TCR repertoire of naive mouse splenocytes. Journal of immunology (Baltimore, Md : 1950). 2000;164(11):5782-7

3. Greiff $V$, et al. Bioinformatic and Statistical Analysis of Adaptive Immune Repertoires. Trends Immunol. 2015;36(11):738-49.

4. Yaari G, Kleinstein SH. Practical guidelines for B-cell receptor repertoire sequencing analysis. Genome Medicine. 2015:1-14.

5. FastQC. Available from: http://www.bioinformatics.babraham.ac.uk/ projects/fastqc.

6. Aronesty, E. ea-utils: Command-line tools for processing biological sequencing data. 2011; Available from: https://github.com/ ExpressionAnalysis/ea-utils.

7. Shugay $M$, et al. Towards error-free profiling of immune repertoires. Nat Methods. 2014;11(6):653-5.

8. Rubelt $F$, et al. Individual heritable differences result in unique cell lymphocyte receptor repertoires of naïve and antigen-experienced cells. Nat Commun. 2016;7:11112.

9. Rounds WH, et al. MSPrecise: A molecular diagnostic test for multiple sclerosis using next generation sequencing. Gene. 2015;572(2):191-7.

10. Rounds $\mathrm{WH}$, et al. The antibody genetics of multiple sclerosis: comparing next-generation sequencing to sanger sequencing. Front Neurol. 2014;5:166

11. Smith TF, Waterman MS. Identification of common molecular subsequences. J Mol Biol. 1981;147(1):195-7.

12. Han A, et al. Linking T-cell receptor sequence to functional phenotype at the single-cell level. Nat Biotechnol. 2014;32(7):684-92.

13. Vander Heiden JA, et al. pRESTO: a toolkit for processing high-throughput sequencing raw reads of lymphocyte receptor repertoires. Bioinformatics (Oxford, England). 2014;30(13):1930-2.

14. Greiff $V$, et al. Quantitative assessment of the robustness of next-generation sequencing of antibody variable gene repertoires from immunized mice. BMC Immunol. 2014;15(1):40

15. Jiang $\mathrm{N}$, et al. Lineage structure of the human antibody repertoire in response to influenza vaccination. Sci Transl Med. 2013;5(171):171ra19.

16. AbStar. [Accessed: June 12, 2017]; Available from: https://github.com/ briney/abstar

17. Thomas N, et al. Decombinator: a tool for fast, efficient gene assignment in T-cell receptor sequences using a finite state machine. Bioinformatics. 2013;29(5):542-50. 
18. Michaeli M, et al. Automated cleaning and pre-processing of immunoglobulin gene sequences from high-throughput sequencing. Front Immunol. 2012;3:386.

19. Safonova $Y$, et al. IgRepertoireConstructor: a novel algorithm for antibody repertoire construction and immunoproteogenomics analysis. Bioinformatics. 2015;31(12):i53-61.

20. Cortina-Ceballos $B$, et al. Reconstructing and mining the $B$ cell repertoire with ImmunediveRsity. MAbs. 2015;7(3):516-24.

21. Zhang W, et al. IMonitor: A Robust Pipeline for TCR and BCR Repertoire Analysis. Genetics. 2015;201(2):459-72.

22. Kuchenbecker $L$, et al. IMSEQ -a fast and error aware approach to immunogenetic sequence analysis. Bioinformatics. 2015;31(18):2963-71.

23. Gerritsen B, et al. RTCR: a pipeline for complete and accurate recovery of $T$ cell repertoires from high throughput sequencing data. Bioinformatics. 2016;32(20):3098-106.

24. Yang $X$, et al. TCRklass: a new K-string-based algorithm for human and mouse TCR repertoire characterization. J Immunol. 2015;194(1):446-54

25. D'Angelo S, et al. The antibody mining toolbox: an open source tool for the rapid analysis of antibody repertoires. MAbs. 2014;6(1):160-72.

26. Fahnrich $\mathrm{A}$, et al. ClonoCalc and ClonoPlot: immune repertoire analysis from raw files to publication figures with graphical user interface. BMC Bioinformatics. 2017:18(1):164.

27. Christley $S$, et al. VDJPipe: a pre-processing pipeline for immune repertoire sequencing data. J Immunol. 2016;196(Suppl 1): p. 209.26.

Submit your next manuscript to BioMed Central and we will help you at every step:

- We accept pre-submission inquiries

- Our selector tool helps you to find the most relevant journal

- We provide round the clock customer support

- Convenient online submission

- Thorough peer review

- Inclusion in PubMed and all major indexing services

- Maximum visibility for your research

Submit your manuscript at www.biomedcentral.com/submit
Biomed Central 